\title{
The Three-Dimensional Angle for Rook Movement within the Restricted Area is its Cosines of the Angle Generated by the Vectors on the Chess Board
}

\author{
M. Laisin, J. E. Okeke, E. I. Chukwuma
}

\begin{abstract}
In this paper, the works of some authors were briefly reviewed with some relevant literature and current state of knowledge pertaining to the three-dimensional proposed rook movement on a chess board within the restricted area were closely studied. We also described some basic terminologies that are helpful in analyzing and studying the rook movements on a three-dimensional chess board. However, we showed that the angle between the vertical and the horizontal rook movement on the same chess board must be $90^{\circ}-[\alpha, \beta, \theta]$. We also showed that $\nabla M$ is a unit vector in the direction of the rook movement Furthermore, the rook movement and the distance from a fixed point $F\left(v_{1}, v_{2}, v_{3}\right)$ to any point $T\left(s_{1}, s_{2}, s_{3}\right)$ for a three-dimensional chess board with the maximum number of arrangements for the $\mathbf{k}$ non-attacking rooks were obtained on an $8 \times 8$ array board. Furthermore, some combinatorial problems were solved by applying generating functions to the rook movement.
\end{abstract}

Index Terms-Chess movements, combinatorial structures, Disjoined chess board in three-dimension, Permutation, three-dimensional structures, r-arrangement, Vectors, Vector generating function

\section{INTRODUCTION}

The study of generating combinatorial structures has a long and distinguishing history. However, the exhaustive listing of elements in relationship to combinatorial structures has one of the first non-trivial problems to be tackled by computer (Knuth 2005). This paper, reviews some of the relevant literature and current state of knowledge pertaining to the three-dimensional proposed rook movement on a chess board within restricted area. It also describes the basic terminologies that are helpful in analyzing and understanding the rook movements on a three-dimensional chess board.

However, Rook/Bishop polynomial has a non-ignorable part to play in the theory of permutations with restricted positions. Laisin\&Uwandu, 2019; Laisin, Okoli, \&Okaa-onwuogu, 2019; LAISIN, 2018; Laisin, 2018; Skoch, 2015; have shown that polynomial of either the rook / bishop on a given board can be generated recursively by applying cell decomposition method of Riordan (Riordan, 1980).

Furthermore, Laisin, 2018; Laisin\&Ndubuisi, 2017; scrutinized, examined and investigated rook movement on a

\footnotetext{
M. Laisin, Department of Mathematics Chukwuemeka Odumegwu Ojukwu University,Anambra State, Nigeria

J. E. Okeke, Department of Mathematics Chukwuemeka Odumegwu Ojukwu University,Anambra State, Nigeria

E. I. Chukwuma. Department of Mathematics Chukwuemeka Odumegwu
} Ojukwu University,Anambra State, Nigeria chess board within restricted area with techniques, such as permutations, combinations, polynomials using generating functions. Also, they determined solutions to the fundamental problems by examining the existence, enumeration and structure of the proposed rook movement on a chess board. Informally, the rook movement on a chess board that are classified into three categories: search, generation, and enumeration (Laisin, 2017).

In-addition, rook theory has a long interesting history arising from permutation problems with restricted positions (Laisin, 2018; Herckman, 2006; Jay \&Haglund, 2000). The theory of rook on Ferrers boards was first developed by Foata and Schutzenderger, 1970 with details on characterization of rook equivalence through bijective proofs. Goldman, Joichi and White 1975 introduced a new face of the rook polynomial of a Ferrers board and gave a combinatorial interpretation of it which showed that it has all integer roots. However, in connections with chromatic polynomials by applying Ferrers board techniques with the rook polynomial which led us to modeling of some binomial theorems (Goldman, Joichi, Reiner, and White, 1977). Also, Goldman, Joichi, and White 1977, studied Orthogonal polynomial while Haglund, 1996, worked on hyper geometric series and so many other large literatures on Ferrers board.

Laisin, 2018; made some generalization of rook polynomials that generalized a classic notion of placing non-taking rooks on a chess board while Jay and Haglund, 2000, worked Ferrers board where rooks are placed on the columns of the board moving from left to right as new rows are created and generalizations on rook polynomial, this leads to a large new combinatorial models' class with connections to sequence polynomial of binomial type and many other models such as permutation of sets and multiset, Bessel polynomials and matching theory, and forest and Abel polynomials.

Furthermore, Jay and Haglund, 2000; focused on working examples of these models, constructing bijections and reasoning with the rook polynomial on a Ferrers board. In fact, the basis of their research was on the notion of an $i$-row creation rook placement. This means that the column where rooks will be placed is chosen first. Then, as non - attacking rooks is placed in the columns from left to right, each time a rook is placed, an $i-$ new rows are created and drawn to the right end and immediately above where the rook is placed. For $i>0$, as rook is being placed, the next rook to be placed has an increased number of possible positions.

The rook polynomials provide a way of counting permutation with restricted positions that was developed by Kaplansky, and Riordan, 1946. Laisin, 2018; Nickolas and Feryal, 2009 generalized these properties and theorems for 2 - dimensional 
rook polynomials. However, more advanced dimensions were partially done for the 3 - dimensional cases (Zindle, 2007).

Now, we shall be focusing on disjoined chess board in three dimensions for non-attacking rooks that generates the sum $90^{\circ}-[\alpha, \beta, \theta]$ as sum of its angles. In addition, we worked on generalization of rook polynomials for a twodimensional and also for a three-dimensional rook polynomial on a disjoined three-dimensional chess board.

\section{A. Basic definitions}

Rook: A rook is a chess piece that moves horizontally or vertically and can take (or capture) a piece if that piece rests on a square in the same row or column as the rook (Laisin, 2018).

a. Board: A board B is an $n \times m$ array of $n$ rows and $m$ columns. When a board has a darkened square, it is said to have a forbidden position.

Rook polynomial: A rook polynomial on a board $\mathrm{B}$, with forbidden positions is denoted as $R(x, B)$, given by

$$
R(x, B)=\sum_{i=1}^{k} r_{i}(B) x^{i}
$$

Where $R(x, B)$ has coefficients $r_{i}(B)$ representing the number of non-capturing rooks on board B. Clearly, we have just one way of not placing a rook. Thus $r_{0}(B)=1$

b. A board B with forbidden positions, is said to be disjoint if the board can be decomposed into two sub-boards $B_{i}: i=1$ and 2 such that, neither $B_{1}$ nor $B_{2}$ share the same row or column.

Fig. 3

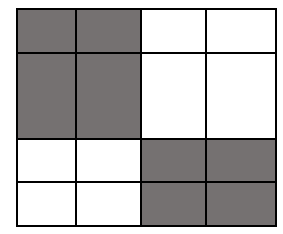

Fig. 4

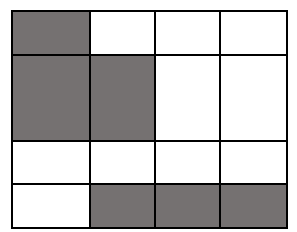

Clearly, fig 3 is disjoint while fig 4 is not.

Boards are invariant, they can be rearranged by swapping rows with rows or by swapping columns with columns. This allows us to attempt to make non-disjoint boards into disjoint boards. Non-taking rooks is to enumerate the number of ways of placing $i$-rooks on a chessboard such that no rook will be captured by any other rook.

\section{B. Basic definitions}

The direction of a vector in three-dimensions is determined by the angles which the vector makes with the three axes of reference

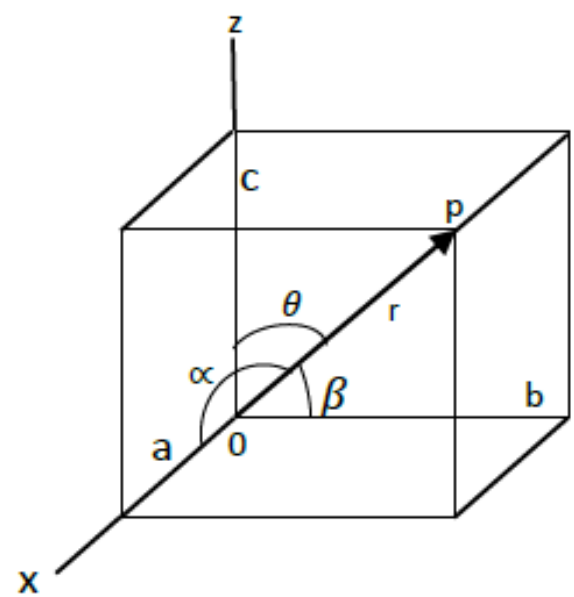

Let $O P=r=a i+b j+c k$. Then $\cos \propto=\frac{a}{r}, \cos \beta=$ $\frac{b}{r}$, and $\cos \theta=\frac{c}{r}$. we have

$a^{2}+b^{2}+c^{2}=r^{2}$ and it follows that $r^{2} \cos ^{2} \propto$

$+r^{2} \cos ^{2} \beta+r^{2} \cos ^{2} \theta=r^{2}$ $\Rightarrow \cos ^{2} \alpha+\cos ^{2} \beta+\cos ^{2} \theta=1$

Thus, if $\cos \propto=\frac{a}{r}, \cos \beta=\frac{b}{r}$, and $\cos \theta=\frac{c}{r}$ and $l=$ $\cos \propto, m=\cos \beta, n=\cos \theta$ then, we have

$$
l^{2}+m^{2}+n^{2}=1
$$

Now, the direction cosines of the vector $O P$ is $\left[l^{2}+m^{2}+\right.$ $\left.n^{2}\right]$

Thus, given the vector $r=a i+b j+c k$, we have that $l=$ $\frac{a}{r}, m=\frac{b}{r}, n=\frac{c}{r}$ and the

$$
|r|=r=\sqrt{a^{2}+b^{2}+c^{2}}
$$

\section{THEOREM (two-dimensional n-disjoint sub-boards)}

If $B$ is a board of darkened squares that decomposes into $n$ disjoint sub-boards $B_{1}, B_{2}, \ldots B_{n}$, then

$$
R(x, B)=R\left(x, B_{1}\right) R\left(x, B_{2}\right) \ldots R\left(x, B_{n}\right) . \text { (Laisin, 2018) }
$$

\section{THEOREM ((two-dimensional Restricted Positions)}

The number of ways to arrange $\mathrm{n}$ objects among $\mathrm{m}$ positions $(m \geq n)$ such order is maintained, when there are restricted positions is

$$
\begin{gathered}
R(x, B)=\sum_{i=0}^{n} \frac{(-1)^{i} r_{i}(B)(m-i n)}{(m n)} \text { where } m \\
=n \quad(\text { Laisin }, 2018)
\end{gathered}
$$

\section{E. THEOREM}

Let $n$ be an even number, and let $S_{n, n}$ denote an $n \times$ $n$ chessboard with the following specifications: for every row $i, i^{t h}$ and $(n+1-i)^{t h}$ positions are restricted. Then $R\left(x, \mathbf{S}_{n, n}\right)=\left(1+4 x+2 x^{2}\right)^{\frac{n}{2}}$.

\section{F. THEOREM (Possible rook movements)}

Let $B$ be a board of darkened squares. Let $R: B \rightarrow[0, \infty]$ be $\left(M, R_{0, \infty}\right)$-possible rook movements. Then, there exist simple rook movement functions $\gamma_{n}, n \in N_{+}$on $B$ such that $\gamma_{n} \uparrow R$. 


\section{G. THEOREM}

Let $B_{m, n, r}$ denote an $m \times n \times r$ chessboard with no restricted positions, and let $s=\min \{m, n, r\}$. Then;

$$
R\left(B_{m, n, r}\right)=\sum_{k=0}^{s}(m k) P_{(n, k)} P_{(r, k)} x^{k},
$$

\section{H. THEOREM}

Consider figure 2.6 as three-dimensional disjoint boards. Then

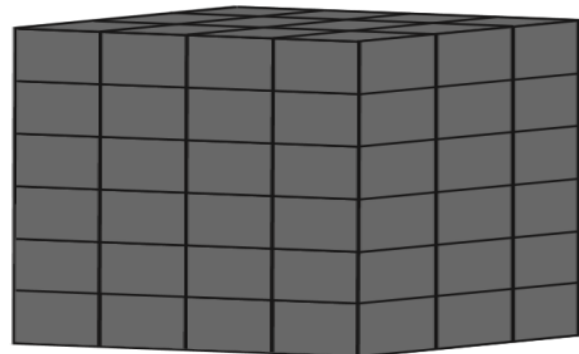

Fig 2.6

The number of ways of placing $\mathrm{k}$ non-attacking rooks on the full $\mathrm{m} \times \mathrm{n}$ board is equal to $(m k)(n k) k !, \forall 0 \leq k \leq \min (m, n) \quad$ (Nickolas and Feryal, 2009)

\section{MAIN RESULTS}

\section{Theorem}

If the movement on a three-dimensional disjoined chess boards is a rook movement, then the angle between the vertical and the horizontal rook movement must be $90^{\circ}-$ $[\alpha, \beta, \theta]$.

\section{Proof}

Consider the direction of a vector in three-dimensions is determined by the angles which the vector makes with the three axes of reference

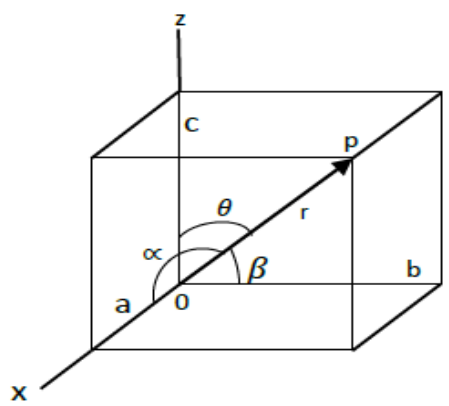

Let $O P=r=a i+b j+c k$. Then $\cos \propto=\frac{a}{r}, \cos \beta=$

$$
\begin{gathered}
\frac{b}{r} \text {, and } \cos \theta=\frac{c}{r} \text {. we have } \\
a^{2}+b^{2}+c^{2}=r^{2}
\end{gathered}
$$

Let the rook movement on a three-dimensional chess board be $r=a i+b j+c k$ for horizontal and vertical rook movement. Then, the angle formed at the horizontal by the rook movement on the three-dimensional disjoined chess board are $\alpha, \beta$ and $\theta$, given as follows; $\alpha=\left(\frac{a}{|(a, b, c)|}\right)$, $\beta=\left(\frac{b}{|(a, b, c)|}\right)$ and $\theta=\left(\frac{c}{|(a, b, c)|}\right)$ respectively. Similarly, the angles formed at the vertical by the rook movement on the three-dimensional disjoined chess board are $\alpha^{*}, \beta^{*}$, and $\gamma^{*}$ respectively. Since, the angle for the horizontal and the vertical rook movement is $90^{\circ}$, then we have

$$
\begin{aligned}
& {[\alpha, \beta, \theta]+\left[\alpha^{*}, \beta^{*}, \theta^{*}\right]=90^{\circ}} \\
& {\left[\alpha^{*}, \beta^{*}, \theta^{*}\right]=90^{\circ}-[\alpha, \beta, \theta]}
\end{aligned}
$$

Thus, it follows that the angle for the horizontal and the vertical rook movement on the restricted area is its cosines of the angle generated by the vectors on the chess board, then it follows that the disjoined chess board are three-dimensional.

\section{THEOREM 2:}

Suppose $\mathrm{M}$ is the rook movement and the distance from a fixed point $F\left(v_{1}, v_{2}, v_{3}\right)$ to any point $T\left(s_{1}, s_{2}, s_{3}\right)$. Then, the $\nabla M$ is a unit vector in the direction of the rook movement $F T=M$.

\section{Proof}

$\mathrm{M}$ is the rook movementthen, $T\left(s_{1}, s_{2}, s_{3}\right)$ is any position to the horizontal/vertical of $F\left(v_{1}, v_{2}, v_{3}\right)$.

Let $m_{F}$ and $m_{T}$ be the rook position vectors for the initial and final rook positions with vectors $v_{1} i+v_{2} j+v_{3} k$ and $s_{1} i+s_{2} j+s_{3} k$ respectively.

Now

$$
\begin{gathered}
M=m_{T}-m_{F} \\
=\left(s_{1} i+s_{2} j+s_{3} k\right)-\left(v_{1} i+v_{2} j+v_{3} k\right) \\
=\left(s_{1}-v_{1}\right) i+\left(s_{2}-v_{2}\right) j+\left(s_{3}-v_{3}\right) k
\end{gathered}
$$

Then, the distance of the rook movement is;

Then,

$$
M=\left[\left(s_{1}-v_{1}\right)^{2}+\left(s_{2}-v_{2}\right)^{2}+\left(s_{3}-v_{3}\right)^{2}\right]^{\frac{1}{2}}
$$

$$
\begin{gathered}
\nabla M=\nabla\left[\left(s_{1}-v_{1}\right)^{2}+\left(s_{2}-v_{2}\right)^{2}+\left(s_{3}-v_{3}\right)^{2}\right]^{\frac{1}{2}} \\
=\frac{\left(s_{1}-v_{1}\right) i+\left(s_{2}-v_{2}\right) j+\left(s_{3}-v_{3}\right) k}{\left[\left(s_{1}-v_{1}\right)^{2}+\left(s_{2}-v_{2}\right)^{2}+\left(s_{3}-v_{3}\right)^{2}\right]^{\frac{1}{2}}} \\
=\frac{M}{M}
\end{gathered}
$$

Thus, it is the unit vector to the rook movement in the dipection $M=F T$.

\section{APPLICATIONS}

\section{Example 4.1}

Determine the maximum number of rooks that are non-attacking on a forbidden chess board and its generating function. Hence, find its rook projection on the vector $i+$ $j+k$.

\section{Solution}

Considering, $B_{1}$ we have for $\mathrm{k}$ non attacking rooks on a 
disjoined chess board as follows;

$$
\begin{aligned}
R\left(x, B_{1}\right)=\sum_{i=0}^{n} & (x)^{i} r_{i}\left(B_{1}\right) \\
& =1+x r_{1}\left(B_{1}\right) \\
& +x^{2} r_{2}\left(B_{1}\right)+\ldots+x^{n} r_{n}\left(B_{1}\right)
\end{aligned}
$$

Since, $B_{1}$ is a two-dimensional chess board, then, the total number of rooks that give a maximum number of rook moves on the forbidden space with non-attacking rooks is as follows;

We have the following rook placements; $r_{0}\left(x, B_{1}\right)=$ $1, r_{1}\left(x, B_{1}\right)=8$ ways, $r_{2}\left(x, B_{1}\right)=21$ ways, $r_{3}\left(x, B_{1}\right)=$ 20 ways, $r_{4}\left(x, B_{1}\right)=5$ ways,

$r_{5}\left(x, B_{1}\right)=4$ ways, $r_{6}\left(x, B_{1}\right)=2$ ways and $r_{7}\left(x, B_{1}\right)=$ 1 way respectively.

However, the two-dimensional rook boards generate the rook function with the generating function.

$$
\begin{gathered}
R\left(x, B_{1}\right)=\sum_{i=0}^{7}(1,8,21,20,5,4,2,1) x^{i} \\
=1+18 x+21 x^{2}+20 x^{3}+5 x^{4}+4 x^{5}+2 x^{6}+x^{7}
\end{gathered}
$$

Thus, $R\left(x, B_{1}\right)$ is the pathway generated by the rook movements for the horizontally and vertically rook movements. Hence, a projection of the vector of $8 i+8 j+$ $8 k$ on the vector $i+j+k$ has a unit vector that is given as $\frac{1}{\sqrt{3}}(i+j+k)$ that gives us a rook projection movement of $\frac{24}{\sqrt{3}}$ units. As required..

\section{CONCLUSION}

The movements polynomials as Rook are very interesting for the both two and three-dimensional cases. Rook polynomials have a variety of applications because they directly relate to permutations with restricted positions. This implies that Rook polynomials can be applied from combinatorial design theory to cryptography.

In this paper we were able to develop the total number of ways to arrange $\mathrm{n}$ rooks among $\mathrm{m}$ positions $(m \geq n)$ and also the construction of rook polynomial that decomposes into 8 -disjoint sub-boards $B_{1}, B_{2}, \ldots B_{8}$ by using an $8 \times 8$ array board. However, we were able to show that the angle between the vertical and the horizontal rook movement must be $90^{\circ}-[\alpha, \beta, \theta]$.

Furthermore, the rook movement and the distance from a fixed point $F\left(v_{1}, v_{2}, v_{3}\right)$ to any point $T\left(s_{1}, s_{2}, s_{3}\right)$ for a three-dimensional chess board with the maximum number of arrangements for the k non-attacking rooks were obtained on an $8 \times 8$ array board.

\section{RECOMMENDATIONS}

Further study can be carried out with the rooks on three-dimensional disjoined boards to determine a general pathway for rook movements on a three- dimensional chess board. In addition, further studies could also examine what would happen to rook polynomials by changing the shape of the boards.

\section{REFERENCES}

[1] D.C. Foata and M,P.Schutzenberger, (1970); on the rook polynomial of Ferrers reactions, colloq. Maths. Janos Bolyai 4, Combinatorial Theory and its applications vol. 2, North - Hall and Amsterdam,, PP. 413-436.

[2] Goldman, J., Joichi, J.T., Reiner, D., and White, D. (1977). Rook Theory ii. Boards of binomial type. SIAM Journal on Applied Mathematics, 31, 617-633.

[3] Goldman, J., Joichi, J.T, and White, D. (1977). Rook Theory iii. Rook polynomials and the achromatic structure of graphs. Journal of Combinatorial Theory, Series B, 25, 135 - 142

[4] Haglund, J. (1996). Rook Theory and hyper geometric series. Advances in Applied Mathematics. 17: 408-459.

[5] Heckman, Christopher, (2006); Rook polynomial N.P. :n.p, n.d. PDF

[6] Jay Goldman, and James Haglund, 2000. Generalized Rook Polynomials. Journal of Combinatorical Theory, Series A91, 509-503 (2000). doi.10. 1006/jcta. 2000. 3113, available online at http: //www.idealibrary.com

[7] Jordan Bell and Brett Stevens, (2009); A survey of known result and research for $\mathrm{n}$ - queens, Discrete Mathematics 309. No 1, 1-31.

[8] Kaplansky-Riordan (1946); Irving Kaplansky, John Riordan, "The problem of the rooks and its applications", Duke Mathematical Journal Volume 13, Number 2 (1946), 259-268.

[9] D.E. Knuth; Fundamental Algorithms, the Art of Computer Programming; Vol. 1 (Second Edition);

[10] Addison-Wesley, Reading, Massachusetts, 1973.

[11] M. Laisin, (2018); Construction of rook polynomials using generating functions and $\mathrm{n} x \mathrm{~m}$ arrays. International Journal of New Technology and Research (IJNTR). ISSN: 2454-4116, Vol. 1, No. 2, Pg. 147-156.

[12] M. Laisin, and W. Uwandu, 2019. The movement Techniques of a Bishop on a Chess Board Generates a Finite Sum of Two Terms. IOSR Journal of Mathematics (IOSR - JM) e - ISSN: 2278 - 5728, P - ISSN: 2319 - 765X. Vol. 15Issue 5 Ser. 1 (Sep - Oct 2019), PP $09-13$. Www.iosrjournal.org

[13] LAISIN, M., (2018). Enumerative techniques for Bishop Polynomials generated by $\Theta^{\wedge} 0$ Board with an $\mathrm{m} \times \mathrm{n}$ array. International Journal of Scientific and Research Publications (IJSRP) 9(1), (ISSN 2250 3153), DOI: http://dx.doi.org/10.29322/IJSRP.9.01.2019.P8599.

[14] Laisin,M., Okoli, O.C., and Okaa - Onwuogu, C.A., (2019). The bishop movement on a chess board is a non - commutative diagonal movement generated by a Laurent monomial.Coou Journal of Physical Science (CJPS), ISSN: 2465 - 6739, Vol. 2, No. 2, Pg. 1 - 6.

[15] Laisin Mark, and F. Ndubuisi, 2017. Combinatorical Applications of Rook Polynomials. Conference of the Faculty of Physical Sciences, ChukwuemekaOdumegwuOjukwu University, Uli. Proceedings of FAPSCON 2017, Uli, Nigeria, http://coou.edu.ng/oer/conference-papers/149

[16] Laisin Mark, (2017); Existence, enumeration and structure of the combinatorial algorithms. Conference of the Faculty of Physical Sciences, ChukwuemekaOdumegwuOjukwu University, Uli. Proceedings of FAPSCON 2017, Uli, Nigeria, http://coou.edu.ng/oer/conference-papers/149

[17] Nicholas Krzywonos, and FeryalAlayont, (2009). Rook Polynomials In Higher Dimensions. Grand Valley State University.

[18] Riordan, J. (1980). An introduction to combinatorial Analysis. New York: Princeton University Press, (Originally published by John Wiley and Sons) Chapman and Hall.

[19] Skoch, S. R., (2015). I Don't Play Chess: A study of chess piece Generating Polynomial. Senior Independent Study Thesis, (P. paper 6559).

[20] Zindle Benjamin (2007) Rook Polynomial for chessboard of two and three Dimensions. School of Mathematical Sciences, Rochester Institute of Technology, New York. 